\title{
Small Molecule Modulation of Voltage Gated Sodium Channels
}

\author{
Vincenzo Carnevale and Michael L. Klein
}

\begin{abstract}
Voltage gated sodium channels are fundamental players in animals physiology. By triggering the depolarization of the lipid membrane they enable generation and propagation of the action potential. The involvement of these channels in numerous pathological conditions makes them relevant target for pharmaceutical intervention. Therefore, modulation of sodium conductance via small molecule binding constitutes a promising strategy to treat a large variety of diseases. However, this approach entails significant challenges: voltage gated sodium channels are complex nanomachines and the details of their workings have only recently started to become clear. Here we review - with emphasis on the computational studies - some of the major milestones in the long-standing search of a quantitative microscopic description of the molecular mechanism and modulation of voltage-gated sodium channels.
\end{abstract}

\section{Introduction: Physiological Role of Voltage Gated Sodium Channels (VGSCs)}

Cells respond to stimuli from the environment by enabling the passage of ions across the plasma membrane, a process that results in the propagation of an electrical signal. Ion channels are the key players of this process, the membranes of excitable cells are studded with a myriad of these integral membrane proteins, which transduce chemical and electrical stimuli into currents of charged chemical species (Hille, 2001). Owing to their pivotal role in cell physiology, a large number of genes encode for ion channels, especially in higher organisms (Harmar et al., 2009). Some of these channels are crucial for the physiology of animals: the emergence of a complex body plan in metazoa implied the necessity to coordinate the response of cells far away from each other and thus the development of a specialized electric network, i.e. the nervous system (Liebeskind, 2011, Liebeskind et al., 2016; Marlow and Arendt, 2014).

Basic building blocks of the nervous system are the neurons, excitable cells with the ability of sustaining the propagation of electric signals with a defined waveform strictly 
preserved throughout the transmission process (Kandel \& Schwartz, 2013). This requirement is satisfied thanks to the coordinated action of distinct types of ion channels, each allowing, at a precise time, the translocation of a specific ionic species (Hille, 2001). Briefly, at resting conditions, the plasma membrane of the neuron is polarized; a perturbation of this resting potential (a depolarization) in a section of a neuron axon causes the activation of two types of channels: first those that enhance this perturbation and then those that counteract it and bring the neuron to its resting condition. Thus the electrical signal, so called action potential, is characterized by two phases: a rising and a falling phase (Elmslie, 2001).

The rising phase of the action potential results from the opening of voltage-gated sodium channels (VGSCs), which decreases the local charge imbalance responsible for the resting potential, thereby generating the rising phase of the action potential (Catterall, 2000; Guy and Seetharamulu, 1986). Thus VGSCs are initiators of the action potential and, as such, they are one of the most critical components of the nervous system. Perhaps not surprisingly, mutations in VGSCs can causative both neuronal hypo- and hyperexcitability and, accordingly, have been found to be associated with inherited syndromes (George, 2005; Mantegazza et al., 2010). Owing to this crucial role played in diseases, VGSCs are among the most important targets of pharmacological agents.

\section{Pharmacology of VGSCs}

Many FDA approved drug molecules target voltage gated sodium channels (VGSCs), usually to reduce cell excitability via suppression of VGSCs activity. The most important examples are: local anesthetics (LA, such as lidocaine), general anesthetics (sevoflurane, isoflurane) anticonvulsants (carmazepine, lamotrigine), and antiarrhythmic drugs (mexiletine). The discovery of these drugs was in most cases serendipitous and thus occurred without a precise knowledge of the molecular target. As a result, these sodium channel modulators typically show weak affinity for the target and poor selectivity toward specific channel subfamilies. Given the ubiquity of VGSCs, non-selective modulators often raise potential risks of severe side effects, thereby limiting the dose-range, the administration routes, or both. This lack of specificity is best discussed by recalling the major structural features of these channels.

\section{Structure of VGSCs}

VGSCs are membrane proteins that contain 24 transmembrane alpha-helices (Catterall, 2000, Noda et al., 1984; Noda et al., 1986). These alpha-helices constitute four homologous, though not identical repeats, conventionally denoted as DI through DIV, each constituted by transmembrane helices (S1 through S6). Sequence analysis indicates that these repeats share a common ancestor with the six-transmembrane-helix domain that characterizes voltage-gated potassium channels (VGPCs), usually referred to as the 6TM domain.

This homology suggests that VGSCs and VGPCs are characterized by the same structural architecture, although in one case (VGPCs) the structure results from the quaternary arrangement of four identical subunits, while in the other (VGSCs) it is the tertiary structure that shows an approximately four-fold symmetry and is referred to as pseudotetramer (Yu \& Catterall, 2004). The recently determined structure of Cav1.1 (Wu et al., 2015), a pseudotetrameric voltage-gated calcium channel closely related to VGSCs, has provided solid experimental validation to this hypthesis. 
This evolutionary relationship between VGSCs and VGPCs raises the hypothesis that the ancestors of the former had the same primary structure as the latter (i.e. a single 6TM domain), before the gene underwent two rounds of duplications (Strong, Chandy, \& Gutman, 1993). This scenario is consistent with the large degree of observed sequence similarity between VGSCs and bacterial voltage-gated sodium selective channels, which show a primary structure constituted by a single 6TM domain assembling in homotetramers.

Similarly to all voltage-gated ion channels, VGSCs show an assembly of four S6 helices, one from each domain, lining the hydrophilic pathway through which conduction of ions occurs. There are two functionally crucial structural elements in this pathway: (i) the gate, that prevents diffusion of ions when the channels is closed thanks to the hindrance of bulky hydrophobic groups; (ii) the selectivity filter, which is selectively permeable to $\mathrm{Na}^{+}$ions (Furini \& Domene 2011; Carnevale et al. 2011; Stock et al. 2013). Four amino acids, one from each domain, constitute the selectivity filter by forming a ring, the so-called DEKA motif, lining the conduction pathway (Heinemann, Terlau, Stühmer, Imoto, \& Numa, 1992).

\section{Molecular Mechanism of VGSCs}

VGSCs open and close in response to a change in polarization of the lipid membrane. Their activation is governed by the same mechanisms used by other members of the voltagegated ion channels superfamily like VGPCs. The structural element responsible for voltage sensitivity is called voltage sensor domain and is constituted by the assembly of the first four transmembrane helices (S1-S4), while the element directly controlling passage of the ions, the gate, is contained within the assembly of the last two transmembrane helices S5 and S6 (McCusker et al., 2012, Payandeh et al., 2011, Shaya et al., 2014; Zhang et al., 2012; Palovcak et al. 2014). This occurs thanks to an allosteric coupling between the conformation of the pore and that of the voltage sensor domain. In particular, the helical segment S4 is characterized by a series of charged residues (almost invariably arginines) showing a periodicity of three amino acids along the sequence. This highly charged secondary structure element is poised to respond to a change in the electrostatic field across the membrane by moving along the membrane normal in a stepwise fashion. Specifically, S4 undergoes a helical screw motion that triggers the opening and closing of the gate in the S6 segment (Amaral et al., 2012, DeCaen et al., 2009, Delemotte et al., 2011, Freites et al., 2012, Henrion et al., 2012, Jensen et al., 2012, Khalili-Araghi et al., 2010 and Vargas et al., 2012).

Several structural studies have highlighted the major mechanical feature of VGPCs: the coupling between the pore domain and the voltage sensor domain regions, which occurs thanks to an intervening amphipathic helix, namely the S4-S5 linker (Long, Tao, Campbell, \& MacKinnon, 2007). This mechanical coupling has been extensively characterized in mutagenesis studies showing that mutating either the S4-S5 linker or the S6 C-term results almost inevitably in severely altered gating properties; importantly, coupling is rescued if compensatory mutations are introduced in the opposing region, an observation that suggests that electromechanical coupling relies on shape and chemical complementarity between these two regions (Labro et al., 2008, Lu et al., 2002, Tristani-Firouzi et al., 2002; Kasimova, 2015; Barber et al. 2012). The transduction of an electrical signal into mechanical work occurs via translation of S4 along the membrane normal, a motion that is 
transmitted to the pore gate by the S4-S5 linker, which acts as a mediator (Blunck \& Batulan, 2012).

Evolutionary analyses suggest that this activation mechanism is a common, universal feature of the voltage-gated channels superfamily (Palovcak et al. 2014). By contrast, a wide variety of mechanisms have been hypothesized for inactivation, which seems to be sub-family specific. Inactivation is a crucial functional feature of VGSCs that enables the generation and transmission of the action potential. Indeed, the activation of VGSCs is controlled by a positive feedback: channel opening, which is driven by membrane depolarization, further depolarizes the membrane due to the influx of $\mathrm{Na}^{+}$ions. This potential instability is controlled by inactivation, which prevents a runaway of the transmembrane potential. From the point of view of thermodynamics, the inactivated state is a stable nonconductive state usually accessible from the activated state (Ahern, 2013, Armstrong and Bezanilla, 1977, Armstrong et al., 1973 and Bezanilla and Armstrong, 1977).

VGSCs inactivation results from a "hinged-lid" mechanism in which a region of the loop between DIII to DIV establishes transient interactions with the pore gate, thereby hindering conduction of ions (Eaholtz et al., 1994, Rohl et al., 1999, Stühmer et al., 1989, Ulbricht, 2005, Vassilev et al., 1988; West et al., 1992).

A set of three conserved residues, namely isoleucine, phenylalanine and methionine (IFM) constitute a distinctive sequence motif from the linker involved in this interaction. On the other hand the residues from the S6 bundle involved in this interaction are still unknown. Even though several S6 residues from DI and DIV cause dramatic changes in inactivation when mutated (McPhee etal., 1994; Wang et al., 2003), their direct involvement in the binding of the lid is unclear. Indeed, mutations necessarily affect simultaneously the processes of activation and inactivation and the resulting kinetic effects might be difficult to interpret. Despite the fact that many details of the inactivation mechanisms are still unclear, the emerging picture suggests that it requires the presence of specific sequence and structural elements. This might have resulted, along evolution, in different pore sequences compared to other VGPCs. Ultimately, the peculiar nature of VGSCs inactivation, which is distinct from that of all the other VGSCs, could explain the specificity of many drugs for the pore domain of VGSCs.

\section{Pore Domain Binders}

Among the drugs binding to the pore domain of VGSCs to exert a modulatory effect on ion conductance, inhalational anesthetics are perhaps the best characterized from the point of view of molecular mechanism (Barber et al. 2014; Covarrubias et al. 2015).

Volatile general anesthetics have been shown to promote inactivation of VGSCs, thereby causing inhibition (Herold et al. 2012; Herold et al. 2009; Horishita et al. 2008; Ouyang et al. 2007; Stadnicka et al. 1999; Duch et al. 1998; Rehberg et al. 1996). In particular, fast inactivation appears to be favored, at steady state, for lower potentials as shown by Ouyang and Hemmings for Nav 1.2, 1.4, and 1.5 (Ouyang et al. 2007). Moreover, distinct halogenated ethers show different abilities to inhibit Nav1.4, possibly as a result of the different interactions established with the channel (Ouyang et al. 2009). For instance, desflurane produce a large left-shift of the steady-state inactivation curve, while halothane and isoflurane give rise to an enhanced current decay together with a slowed recovery from inactivation. 
In spite of the limited structural information available for eukaryotic VGSCs, several xray crystallography studies have focused on the class of prokaryotic $\mathrm{Na}^{+}$-selective voltagegated ion channels (Payandeh et al. 2011; Bagnéris et al. 2015; Payandeh et al. 2012). Extensive phylogenetic analyses have then ascertained the evolutionary relatedness of these channels with VGSCs (Kasimova et al. 2016). Moreover, several functional and structural studies have confirmed that these channels show structural features and pharmacological profiles consistent with VGSCs (Ouyang et al. 2007; Charalambous et al. 2011; Kuzmenkin et al. 2004; Bagnéris et al. 2014; Richardson et al. 2006). Molecular simulations based on the "flooding" protocol performed on a homology-derived model of one of these channels, namely NaChBac, showed that isoflurane and sevoflurane bind to the channel at four distinct locations (Barber et al. 2014; Raju et al. 2013). The first binding site is in the pore of the channel, a location that is consistent with a pore-blocking mechanism of inhibition. Strikingly, this binding site can be reached by the ligands even when the pore gate is closed and thus the aqueous compartment at the intracellular side is not connected to the channel lumen. Indeed, the so-called "fenestrations", connecting the interior of the pore to the hydrophobic section of the membrane enable diffusion to the binding site and are likely to be the hydrophobic drug access pathway hypothesize several decades ago (Hille 1977). These fenestrations host the second set of binding sites, located at the entrance of these non-polar tunnels. Another set of binding sites is detected in a cavity lined by the S4-S5 linker and the S6 helix bundle. This cavity, which is mostly present in the open state, contains several hydrophobic side chains from the S6 helices; the resulting polarity of the pocket is ideal to accommodate the mostly hydrophobic character of halogenated general anesthetics. The final set of binding sites is found at the interfaces between subunits in pockets that surround the selectivity filter. The cavities are lined by the amino acids of the so-called pore-turret and of the pore-helix. Importantly, these binding sites are directly accessible from the extracellular milieu. These sites are characterized by a marked state-dependent affinity and thus can potentially be associated with allosteric modulation of the channel. In fact, binding to the sites lining the selectivity filter is favorable only in the activated state, while binding to the fenestrations can occur only in the closed state. Importantly, free energy perturbation based affinity calculations indicate that these binding sites are characterized by submillimolar dissociation constants, thus binding is likely to occur at physiological concentrations. Accordingly, in a combined computational and NMR investigation, two of these sites were unambiguously experimentally confirmed (Kinde et al., 2016). However, the lack of systematic in vivo mutagenesis studies does not allow us to draw strong conclusions on the relevance of these sites in mediating the drug induced condition of general anesthesia.

\section{Future Directions: Voltage Sensor Binders}

The promiscuity of local anesthetics for distinct sub-families stems from the strong sequence conservation characterizing the pore domain: genomic surveys have highlighted several immutable residues, arguably a constraint imposed by a finely tuned gating mechanism (DeCaen et al. 2008). A viable mechanism to modulate VGSCs in a selective fashion is exemplified by neurotoxins from the venom of animals (Cestèle et al. 1998). These peptides have evolved to elicit a specific reaction in the host organism, e.g. immobilization of prey, therefore their modulation capability is usually tailored on a specific class of channels. Toxins have a variety of binding sites: some target the pore 
domain, while others the voltage sensors. Examples are: D-conotoxins (Heinemann et al. 2007), which bind DIV S3-S4 and slows down inactivation and scorpion b-toxins, which bind to DII-VSD. The interesting aspect of these VSD binders is that they target a channel region that is extremely variable. Indeed, to properly sustain the uprising phase of the action potential, each VSD from a given VGSCs has undergone functional specialization during evolution: DIII-VSD and DII-VSD activate at lower voltages than DI-VSD and even more depolarized potentials are required to activate DIV-VSD, which triggers the transition toward a non-conductive state of the channel called inactivated state. Specifically, HWTX-IV toxin was shown to stabilize the resting state of DII-VSD of Nav1.7, thereby inhibiting the channel (Shi et al. 2012; Xiao et al. 2010). The structural determinants of the toxin-channel interaction could be in principle used to design small molecule binders of the resting state of Nav1.7 DII-VSD. While such molecules would certainly address effectively the medical need of pain control, reports of VSDs binders have been so far scanty.

This pharmacological strategy is seemingly neglected because of the conceptual obstacles entailed by this peculiar drug design process. Some of the major issues are: absence of an obviously defined pocket for binding, involvement of waters and lipids, strong dependence of the structure on the conformational state, and possibly small interaction surface for anchoring the ligand. In spite of these challenges recent advances in protein engineering hold promise to make voltage sensor domain a viable target for drug design as recently shown by Payandeh and coworkers (Ahuja et al. 2015). By introducing the voltage sensor domain of Nav1.7 into an engineered bacterial channel, this crystallographic study reveals, for the first time, the molecular interactions between a drug-like molecule and a voltage sensor domain. In particular, the authors showed that an aryl sulfonamide inhibitor is able to bind with high affinity by directly engaging the fourth gating charge residue (R4), thereby hindering the conformational transition of the voltage sensor required for activation.

\section{Acknowledgements}

This work was supported by the National Institutes of Health, National Institute for General Medical Sciences [P01 GM55876 (M.L.K.)] and by the National Science Foundation through (i) grant ACI-1614804 (V.C. and M.L.K.) and (ii) major research instrumentation grant number CNS-09-58854.

\section{Figures}

A

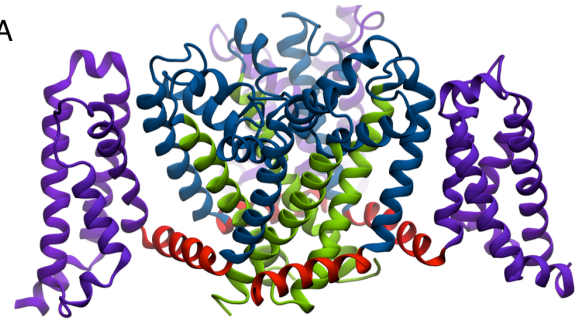

B

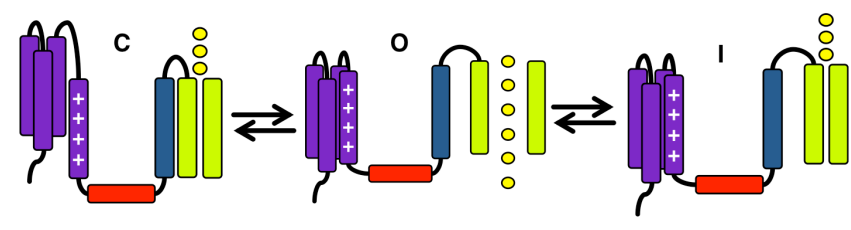

Figure 1. Cartoon representation of the structure and mechanism of voltage gated sodium channels. The first four transmembrane (TM) helices (S1 through S4 from each subunit) constitute the voltage sensor domain (purple). The pore domain, instead, comprises two TM helices, S5 and S6 (blue and yellow, respectively. The pore and voltage sensor domains 
are connected by the linker domain (red). (B) The gating mechanism of VGSCs requires a coordinated motion of the voltage sensor and pore domains. Under depolarizing conditions, the positively charged S4 helix translates along the bilayer normal causing the splaying of the S6 helix bundle. The channel thus transitions from the closed (C) to the open state (0). The latter is unstable and decays to the inactivated state (I), a nonconductive state characterized by an activated voltage sensor domain and a closed pore domain.
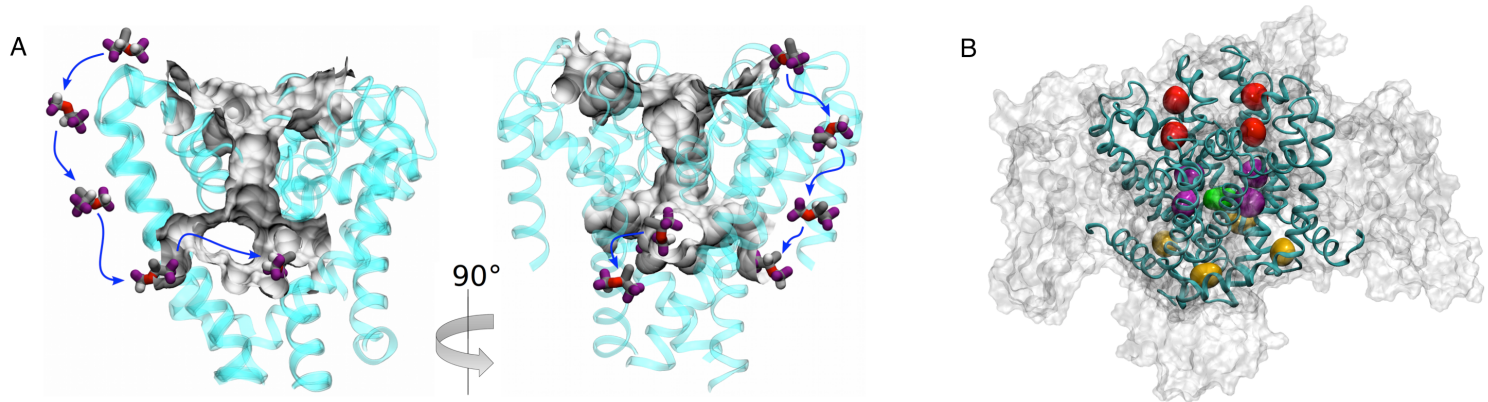

Figure 2. Binding of the inhalational general anesthetic isoflurane to the voltage gated sodium selective bacterial channel NaChBac. A) An isoflurane molecule diffusing in and out of the pore of NaChBac through adjacent fenestrations. The channel cavity is rendered as a solid grey surface. B) The three binding sites identified by clustering analysis: extracellular site (red), linker site (yellow) and cavity site (purple/green).

\section{References}

Ahern, C.A. 2013. What activates inactivation? J. Gen. Physiol.. 142:97-100. doi:10.1085/jgp.201311046.

Amaral, C., V. Carnevale, M.L. Klein, and W. Treptow. 2012. Exploring conformational states of the bacterial voltage-gated sodium channel NavAb via molecular dynamics simulations. Proc. Natl. Acad. Sci. U. S. A.. 109:21336-21341. doi:10.1073/pnas.1218087109.

Armstrong, C.M., and F. Bezanilla. 1977. Inactivation of the sodium channel. II. Gating current experiments. J. Gen. Physiol.. 70:567-590.

Armstrong, C.M., F. Bezanilla, and E. Rojas. 1973. Destruction of Sodium Conductance Inactivation in Squid Axons Perfused with Pronase. J. Gen. Physiol.. 62:375-391.

Bagnéris, C., P.G. DeCaen, C.E. Naylor, D.C. Pryde, I. Nobeli, D.E. Clapham, and B.A. Wallace. 2014. Prokaryotic NavMs channel as a structural and functional model for eukaryotic sodium channel antagonism. Proc. Natl. Acad. Sci.. 111:8428-8433. doi:10.1073/pnas.1406855111. 
Barber, A.F., V. Carnevale, M.L. Klein, R.G. Eckenhoff, and M. Covarrubias. 2014. Modulation of a voltage-gated $\mathrm{Na}+$ channel by sevoflurane involves multiple sites and distinct mechanisms. Proc. Natl. Acad. Sci.. 111:6726-6731. doi:10.1073/pnas.1405768111.

Barber, A.F., V. Carnevale, S.G. Raju, C. Amaral, W. Treptow, and M.L. Klein. 2012. Hingebending motions in the pore domain of a bacterial voltage-gated sodium channel. Biochim. Biophys. Acta BBA 1818:2120-2125. doi:10.1016/j.bbamem.2012.05.002.

Bezanilla, F., and C.M. Armstrong. 1977. Inactivation of the sodium channel. I. Sodium current experiments. J. Gen. Physiol.. 70:549-566.

Blunck, R., and Z. Batulan. 2012. Mechanism of electromechanical coupling in voltage-gated potassium channels. Front. Pharmacol.. 3:166. doi:10.3389/fphar.2012.00166.

Carnevale, V., W. Treptow, and M.L. Klein. 2011. Sodium ion binding sites and hydration in the lumen of a bacterial ion channel from molecular dynamics simulations. J. Phys. Chem. Lett.. 2:2504-2508.

Catterall, W.A. 2000. From ionic currents to molecular mechanisms: the structure and function of voltage-gated sodium channels. Neuron. 26:13-25.

Cestèle, S., Y. Qu, J.C. Rogers, H. Rochat, T. Scheuer, W.A. Catterall, Voltage Sensor-Trapping: Enhanced Activation of Sodium Channels by ?-Scorpion Toxin Bound to the S3-S4 Loop in Domain II. Neuron 1998, 21, 919-931.

Charalambous, K., B.A. Wallace NaChBac: the long lost sodium channel ancestor Biochemistry, 50 (2011), pp. 6742-6752

Covarrubias, M., A.F. Barber, V. Carnevale, W. Treptow, and R.G. Eckenhoff. 2015. Mechanistic insights into the modulation of voltage-gated ion channels by inhaled anesthetics. Biophys. J.. in press.

DeCaen, P. G., V. Yarov-Yarovoy, Y. Zhao, T. Scheuer, W.A. Catterall, Disulfide Locking a Sodium Channel Voltage Sensor Reveals Ion Pair Formation during Activation. Proc. Natl. Acad. Sci. 2008, 105, 15142-15147.

DeCaen, P.G., V. Yarov-Yarovoy, E.M. Sharp, T. Scheuer, and W.A. Catterall. 2009. Sequential formation of ion pairs during activation of a sodium channel voltage sensor. Proc. Natl. Acad. Sci. U. S. A.. 106:22498-22503. doi:10.1073/pnas.0912307106.

* An in depth investigation of the residue-residue contacts characterizing each stage of the activation ladder of the voltage sensor domain of a bacterial sodium selective channel.

Delemotte, L., M. Tarek, M.L. Klein, C. Amaral, and W. Treptow. 2011. Intermediate states of the Kv1.2 voltage sensor from atomistic molecular dynamics simulations. Proc. Natl. Acad. Sci. U. S. A.. 108:6109-6114. doi:10.1073/pnas.1102724108. 
Duch, D.S. Rehberg, B. Vysotskaya T.N. Volatile anesthetics significantly suppress central and peripheral mammalian sodium channels Toxicol. Lett., 100-101 (1998), pp. 255-263

Eaholtz, G., T. Scheuer, and W.A. Catterall. 1994. Restoration of inactivation and block of open sodium channels by an inactivation gate peptide. Neuron. 12:1041-1048.

Elmslie, K.S. 2001. Action Potential: Ionic Mechanisms. In eLS. John Wiley \& Sons, Ltd.

Freites, J.A., E.V. Schow, S.H. White, and D.J. Tobias. 2012. Microscopic Origin of Gating Current Fluctuations in a Potassium Channel Voltage Sensor. Biophys. J.. 102:L44-L46. doi:10.1016/j.bpj.2012.04.021.

Furini, S., and C. Domene. 2012. On Conduction in a Bacterial Sodium Channel. PLOS Comput Biol. 8:e1002476. doi:10.1371/journal.pcbi.1002476.

** An accurate characterization of the conduction mechanism and of the determinants of selectivity of a bacterial sodium selective channel.

George, A.L. 2005. Inherited disorders of voltage-gated sodium channels. J. Clin. Invest.. 115:1990-1999. doi:10.1172/JCI25505.

Goldschen-Ohm M.P., Chanda B. Probing gating mechanisms of sodium channels using pore blockers Handbook Exp. Pharmacol., 221 (2014), pp. 183-201

Guy, H.R., and P. Seetharamulu. 1986. Molecular model of the action potential sodium channel. Proc. Natl. Acad. Sci. U. S. A.. 83:508-512.

Harmar, A.J., R.A. Hills, E.M. Rosser, M. Jones, O.P. Buneman, D.R. Dunbar, S.D. Greenhill, V.A. Hale, J.L. Sharman, T.I. Bonner, W.A. Catterall, A.P. Davenport, P. Delagrange, C.T. Dollery, S.M. Foord, G.A. Gutman, V. Laudet, R.R. Neubig, E.H. Ohlstein, R.W. Olsen, J. Peters, J.-P. Pin, R.R. Ruffolo, D.B. Searls, M.W. Wright, and M. Spedding. 2009. IUPHAR-DB: the IUPHAR database of G protein-coupled receptors and ion channels. Nucleic Acids Res.. 37:D680D685. doi:10.1093/nar/gkn728.

Heinemann, S. H.; Leipold, E. Conotoxins of the O-Superfamily Affecting Voltage-Gated Sodium Channels. Cell. Mol. Life Sci. 2007, 64, 1329-1340.

Heinemann, S.H., H. Terlau, W. Stühmer, K. Imoto, and S. Numa. 1992. Calcium channel characteristics conferred on the sodium channel by single mutations. Nature. 356:441-443. doi:10.1038/356441a0.

Henrion, U., J. Renhorn, S.I. Börjesson, E.M. Nelson, C.S. Schwaiger, P. Bjelkmar, B. Wallner, E. Lindahl, and F. Elinder. 2012. Tracking a complete voltage-sensor cycle with metal-ion bridges. Proc. Natl. Acad. Sci.. doi:10.1073/pnas.1116938109. 


\section{** Insightful combined computational and experimental investigation of the voltage sensor domain dynamics. It is one of the most accurate sources thus far of structural information concerning the activation conformational transition.}

Herold, K.F. Hemmings H.C. Jr. Sodium channels as targets for volatile anesthetics Front. Pharmacol., 3 (2012), p. 50

Herold, K.F. Nau, C. Hemmings H.C. Jr., et al. Isoflurane inhibits the tetrodotoxin-resistant voltage-gated sodium channel Nav1.8 Anesthesiology, 111 (2009), pp. 591-599

* This paper demonstrates that volatile general anesthetics have a modulatory effect on voltage-gated sodium channels

Hille B. Local anesthetics: hydrophilic and hydrophobic pathways for the drug-receptor reaction J. Gen. Physiol., 69 (1977), pp. 497-515

Hille, B. 2001. Ion Channels of Excitable Membranes, Third Edition. 3rd Edition edition. Sinauer Associates, Sunderland, Mass. $814 \mathrm{pp}$.

Horishita, T. Eger E.I. 2nd, Harris R.A. The effects of volatile aromatic anesthetics on voltage-gated $\mathrm{Na}+$ channels expressed in Xenopus oocytes Anesth. Analg., 107 (2008), pp. $1579-1586$

Jensen, M.Ø., V. Jogini, D.W. Borhani, A.E. Leffler, R.O. Dror, and D.E. Shaw. 2012. Mechanism of voltage gating in potassium channels. Science. 336:229-233. doi:10.1126/science.1216533.

Kandel, E., and J. Schwartz. 2013. Principles of Neural Science, Fifth Edition. McGraw Hill Professional. $1761 \mathrm{pp}$.

Kinde, MN, Bondarenko, V., Granata D., Bu W., Grasty WC., Loll PJ, Carnevale V, Klein ML, Eckenhoff RG, Tang P, Xu Y. Fluorine-19 NMR and computational quantification of isoflurane binding to the voltage-gated sodium channel NaChBac PNAS 2016113 (48) 13762-13767, doi:10.1073/pnas.1609939113

Khalili-Araghi, F., V. Jogini, V. Yarov-Yarovoy, E. Tajkhorshid, B. Roux, and K. Schulten. 2010. Calculation of the gating charge for the Kv1.2 voltage-activated potassium channel. Biophys. J.. 98:2189-2198. doi:10.1016/j.bpj.2010.02.056.

Kuzmenkin, A. Bezanilla, F. Correa A.M. Gating of the bacterial sodium channel, NaChBac: voltage-dependent charge movement and gating currents J. Gen. Physiol., 124 (2004), pp. 349-356

Labro, A.J., A.L. Raes, A. Grottesi, D.V. Hoorick, M.S.P. Sansom, and D.J. Snyders. 2008. Kv Channel Gating Requires a Compatible S4-S5 Linker and Bottom Part of S6, Constrained by Non-interacting Residues. J. Gen. Physiol.. 132:667-680. doi:10.1085/jgp.200810048. 
Liebeskind, B.J. 2011. Evolution of sodium channels and the new view of early nervous system evolution. Commun. Integr. Biol.. 4:679-683.

Liebeskind, B.J., D.M. Hillis, and H.H. Zakon. 2011. Evolution of sodium channels predates the origin of nervous systems in animals. Proc. Natl. Acad. Sci.. 108:9154-9159. doi:10.1073/pnas.1106363108.

Liebeskind, B.J., D.M. Hillis, H.H. Zakon, and H.A. Hofmann. 2016. Complex Homology and the Evolution of Nervous Systems. Trends Ecol. Evol.. 31:127-135. doi:10.1016/j.tree.2015.12.005.

Long, S.B., X. Tao, E.B. Campbell, and R. MacKinnon. 2007. Atomic structure of a voltagedependent $\mathrm{K}+$ channel in a lipid membrane-like environment. Nature. 450:376-382. doi:10.1038/nature06265.

Lu, Z., A.M. Klem, and Y. Ramu. 2002. Coupling between voltage sensors and activation gate in voltage-gated K+ channels. J. Gen. Physiol.. 120:663-676.

M.A. Kasimova, M.A. Zaydman, J. Cui, M. Tarek PIP2-dependent coupling is prominent in Kv7.1 due to weakened interactions between S4-S5 and S6 Scientific reports, 5 (2015), p. 7474 http://dx.doi.org/10.1038/srep07474

Mantegazza, M., G. Curia, G. Biagini, D.S. Ragsdale, and M. Avoli. 2010. Voltage-gated sodium channels as therapeutic targets in epilepsy and other neurological disorders. Lancet Neurol.. 9:413-424. doi:10.1016/S1474-4422(10)70059-4.

Marlow, H., and D. Arendt. 2014. Evolution: Ctenophore Genomes and the Origin of Neurons. Curr. Biol.. 24:R757-R761. doi:10.1016/j.cub.2014.06.057.

McCusker, E.C., C. Bagnéris, C.E. Naylor, A.R. Cole, N. D’Avanzo, C.G. Nichols, and B.A. Wallace. 2012. Structure of a bacterial voltage-gated sodium channel pore reveals mechanisms of opening and closing. Nat. Commun.. 3:1102. doi:10.1038/ncomms2077.

McPhee, J.C., D.S. Ragsdale, T. Scheuer, and W.A. Catterall. 1994. A mutation in segment IVS6 disrupts fast inactivation of sodium channels. Proc. Natl. Acad. Sci. U. S. A.. 91:1234612350.

Noda, M., S. Shimizu, T. Tanabe, T. Takai, T. Kayano, T. Ikeda, H. Takahashi, H. Nakayama, Y. Kanaoka, N. Minamino, K. Kangawa, H. Matsuo, M.A. Raftery, T. Hirose, S. Inayama, H. Hayashida, T. Miyata, and S. Numa. 1984. Primary structure of Electrophorus electricus sodium channel deduced from cDNA sequence. Nature. 312:121-127. doi:10.1038/312121a0.

Noda, M., T. Ikeda, H. Suzuki, H. Takeshima, T. Takahashi, M. Kuno, and S. Numa. 1986. Expression of functional sodium channels from cloned cDNA. Nature. 322:826-828. doi:10.1038/322826a0. 
Oelstrom, K., M.P. Goldschen-Ohm, M. Holmgren, and B. Chanda. 2014. Evolutionarily conserved intracellular gate of voltage-dependent sodium channels. Nat. Commun.. 5:3420. doi:10.1038/ncomms4420.

OuYang, W. Hemmings H.C. Jr.Isoform-selective effects of isoflurane on voltage-gated Na+ channels Anesthesiology, 107 (2007), pp. 91-98

Ouyang, W. Herold, K.F. Hemmings H.C. Jr. Comparative effects of halogenated inhaled anesthetics on voltage-gated $\mathrm{Na}+$ channel function Anesthesiology, 110 (2009), pp. 582590

Ouyang, W. Jih T.Y., Hemmings H.C. Jr., et al. Isoflurane inhibits NaChBac, a prokaryotic voltage-gated sodium channel J. Pharmacol. Exp. Ther., 322 (2007), pp. 1076-1083

Palovcak, E., L. Delemotte, M.L. Klein, and V. Carnevale. 2014. Evolutionary imprint of activation: the design principles of VSDs. J. Gen. Physiol.. 143:145-156. doi:10.1085/jgp.201311103.

Payandeh J., Gamal El-Din T.M., Catterall W.A., et al. Crystal structure of a voltage-gated sodium channel in two potentially inactivated states Nature, 486 (2012), pp. 135-139

Payandeh, J. Scheuer, T. Catterall W.A., et al. The crystal structure of a voltage-gated sodium channel Nature, 475 (2011), pp. 353-358

** First structural determination of a voltage-gated sodium selective channel. It provides unexpected insight on the structure of the selectivity filter and the presence of hydrophobic pathways (fenestrations) connecting the channel pore to the hydrophobic region of the lipid bilayer.

Payandeh, J., T. Scheuer, N. Zheng, and W.A. Catterall. 2011. The crystal structure of a voltage-gated sodium channel. Nature. 475:353-358. doi:10.1038/nature10238.

Raju S.G., Barber A.F., Carnevale V., et al. Exploring volatile general anesthetic binding to a closed membrane-bound bacterial voltage-gated sodium channel via computation PLOS Comput. Biol., 9 (2013), p. e1003090

Rehberg, B. Xiao, Y.H. Duch D.S. Central nervous system sodium channels are significantly suppressed at clinical concentrations of volatile anesthetics Anesthesiology, 84 (1996), pp. 1223-1233 discussion 27A

** First clear experimental indication that sodium channels can potentially be implicated in the mechanism of general anesthesia.

Richardson J., Blunck R., Correa A.M., et al. Distance measurements reveal a common topology of prokaryotic voltage-gated ion channels in the lipid bilayer Proc. Natl. Acad. Sci. USA, 103 (2006), pp. 15865-15870 
Rohl, C.A., F.A. Boeckman, C. Baker, T. Scheuer, W.A. Catterall, and R.E. Klevit. 1999. Solution structure of the sodium channel inactivation gate. Biochemistry (Mosc.). 38:855-861. doi:10.1021/bi9823380.

Shaya, D., F. Findeisen, F. Abderemane-Ali, C. Arrigoni, S. Wong, S.R. Nurva, G. Loussouarn, and D.L. Minor Jr. 2014. Structure of a Prokaryotic Sodium Channel Pore Reveals Essential Gating Elements and an Outer Ion Binding Site Common to Eukaryotic Channels. J. Mol. Biol.. 426:467-483. doi:10.1016/j.jmb.2013.10.010.

Shih, A. Y.; Bembenek, S.; Minassian, N.; Neff, R.; Liu, Y.; Flinspach, M.; Edavettal, S.; Wu, N.; Maher, M.; Wickenden, A.; et al. Functional Studies of Interaction Between Huwentoxin-IV and Voltage-Gated Sodium Channel Nav1.7. Biophys. J. 2012, 102, 324a.

Stadnicka, A. Kwok, W.M. Bosnjak Z.J., et al. Effects of halothane and isoflurane on fast and slow inactivation of human heart hH1a sodium channels Anesthesiology, 90 (1999), pp. 1671-1683

Stock, L. Souza, C. Treptow W. Structural basis for activation of voltage-gated cation channels Biochemistry, 52 (2013), pp. 1501-1513

Stock, L., L. Delemotte, V. Carnevale, W. Treptow, and M.L. Klein. 2013. Conduction in a biological sodium selective channel. J. Phys. Chem. B. 117:3782-3789.

Strong, M., K.G. Chandy, and G.A. Gutman. 1993. Molecular evolution of voltage-sensitive ion channel genes: on the origins of electrical excitability. Mol. Biol. Evol.. 10:221-242.

Stühmer, W., F. Conti, H. Suzuki, X. Wang, M. Noda, N. Yahagi, H. Kubo, and S. Numa. 1989. Structural parts involved in activation and inactivation of the sodium channel. Nature. 339:597-603. doi:10.1038/339597a0.

Tristani-Firouzi, M., J. Chen, and M.C. Sanguinetti. 2002. Interactions between S4-S5 linker and S6 transmembrane domain modulate gating of HERG K+ channels. J. Biol. Chem.. 277:18994-19000. doi:10.1074/jbc.M200410200.

Ulbricht, W. 2005. Sodium Channel Inactivation: Molecular Determinants and Modulation. Physiol. Rev.. 85:1271-1301. doi:10.1152/physrev.00024.2004.

Vargas, E., V. Yarov-Yarovoy, F. Khalili-Araghi, W.A. Catterall, M.L. Klein, M. Tarek, E. Lindahl, K. Schulten, E. Perozo, F. Bezanilla, and B. Roux. 2012. An emerging consensus on voltage-dependent gating from computational modeling and molecular dynamics simulations. J. Gen. Physiol.. 140:587-594. doi:10.1085/jgp.201210873.

Vassilev, P.M., T. Scheuer, and W.A. Catterall. 1988. Identification of an intracellular peptide segment involved in sodium channel inactivation. Science. 241:1658-1661. doi:10.1126/science.2458625. 
Wang, S.-Y., K. Bonner, C. Russell, and G.K. Wang. 2003. Tryptophan Scanning of D1S6 and D4S6 C-Termini in Voltage-Gated Sodium Channels. Biophys. J.. 85:911-920.

Wu, J., Z. Yan, Z. Li, C. Yan, S. Lu, M. Dong, and N. Yan. 2015. Structure of the voltage-gated calcium channel Cav1.1 complex. Science. 350:aad2395. doi:10.1126/science.aad2395.

Xiao, Y.; Blumenthal, K.; Jackson, J. O.; Liang, S.; Cummins, T. R. The Tarantula Toxins ProTxII and Huwentoxin-IV Differentially Interact with Human Nav1.7 Voltage Sensors to Inhibit Channel Activation and Inactivation. Mol. Pharmacol. 2010, 78, 1124-1134.

Yu, F.H., and W.A. Catterall. 2004. The VGL-Chanome: A Protein Superfamily Specialized for Electrical Signaling and Ionic Homeostasis. Sci. Signal.. 2004:re15. doi:10.1126/stke.2532004re15.

Zhang, X., W.L. Ren, P. DeCaen, C.Y. Yan, X. Tao, L. Tang, J.J. Wang, K. Hasegawa, T. Kumasaka, J.H. He, J.W. Wang, D.E. Clapham, and N. Yan. 2012. Crystal structure of an orthologue of the NaChBac voltage-gated sodium channel. Nature. 486:130-134. doi:10.1038/nature11054. 